\title{
Rat pups are reinforcing to the maternal rat: Role of sensory cues
}

\author{
JANE E. MAGNUSSON and ALISON S. FLEMING \\ Erindale College, University of Toronto, Mississauga, Ontario, Canada
}

\begin{abstract}
The present studies were undertaken to investigate the involvement of sensory cues in the expression of maternal behaviors and in the reinforcing properties of pups, reflected in performance on a conditioned place preference task. Postpartum animals were either able to interact with pups in one of two novel environments or were prevented from doing so by placing pups in a perforated Plexiglas cube. On alternate days, mothers were exposed to a second environment in the absence of pups. When the mothers tested for the development of a conditioned place preference, it was found that the group permitted pup interaction preferred the pup-paired box and that the no-interaction (distal) group did not. The role of the primary olfactory system was investigated by rinsing the nares of animals with either a $5 \%$ zinc sulphate spray to render the animal hyposmic or an air control and then permitting the animals to interact with pups in one of two novel environments. There were no differences between groups in maternal responding. However, when the mothers were tested for the development of a preference for the pup- paired box, it was found that while the air control animals preferred the pup-paired box, the anosmic zinc sulphate animals did not. To investigate the involvement of somatosensory cues, the ventral or perioral surfaces of animals were treated with either an anesthetic cream or a control cream prior to exposure to pups. Testing for maternal behaviors revealed that there were no differences between anesthetized groups and their controls. However, when the animals were tested for the development of a conditioned place preference, it was found that while both the ventral and perioral control groups preferred the pup-paired box, the ventral and the perioral anesthetized groups did not. Taken together, these studies demonstrate that several modalities involved in the interaction between pups and mothers contribute to the pups' ability to be a reinforcing stimulus to the mother. The stimulation that postpartum mothers receive via these modalities is complex, and the disruption of any of them can alter the reinforcing capabilities of pups.
\end{abstract}

Pups are powerful reinforcers to the maternal animal (Fleming, Korsmit, \& Deller, 1994). If maternal females are pup deprived for a $23-h$ period and are then presented with pups in a new environment for a 1-h exposure period, at test they show a preference for the environment that was previously paired with the pups over the environment not paired with pups (Fleming et al., 1994).

The dam's behavior during the exposure or training phase is highly predictive of whether a conditioned place preference (CPP) will develop (Fleming et al., 1994). If dams have not had sufficient maternal experience prior to the postpartum training phase and do not actually crouch over the pups during the paired exposure period, a preference will not develop. A preference also does not develop if the training phase is shortened from 4 to 2 days, if the daily exposure period is shortened from 60 to $30 \mathrm{~min}$, or if nonmaternal virgins are tested. The aspects of the behavioral interaction that are important for pups

This research was supported by an NSERC operating grant to A.S.F. Many thanks to Maureen Patchett for her help with the manuscript, and to Marc Korsmit for his enlightening ideas. Correspondence should be addressed to A. S. Fleming, Department of Psychology, Erindale College, University of Toronto, Mississauga, ON, Canada L5L 1C6. (email: afleming@credit.erin or fleming@psych.utoronto.ca). to acquire reinforcing properties adequate to sustain a CPP constitute the focus of this paper.

The maternal animal is multiply stimulated when she interacts with pups (Beach \& Jaynes, 1956; Stern, 1989); she responds to their ultrasonic cries when they are displaced from the nest or are disturbed in the nest (Allin \& Banks, 1972; Brewster \& Leon, 1980; Brouette-Lahlou, Vernet-Maury, \& Vigouroux, 1992). She retrieves pups after she first touches and mouthes pups, stimulating somatosensory receptors in her perioral/mouth region (Kenyon, Cronin, \& Keeble, 1983; Stern \& Johnson, 1989; Stern \& Kolunie, 1989). Once pups are in the nest, she licks the pups in part in response to their odors, whether natural or artificially applied (Brouette-Lahlou, VernetMaury, \& Chanel, 1991). And she adopts a high-arch lactating posture over the pups when they attach to her teats and stimulate her ventral region (Stern, Dix, Bellomo, \& Thramann, 1992; Stern, Dix, Pointex, \& Thramann, 1990). Each modality, with the possible exception of vision, is thus activated when the dam responds nurturantly to her litter.

Many of the same cues may also be involved in the longterm maintenance of maternal behavior after the initial period when the parturitional hormones are active; they may also be involved in the long-term retention of responsiveness after a period of separation from pups 
(Bridges, 1975, 1977; Cohen \& Bridges, 1981; Orpen \& Fleming, 1987). If dams are prevented from touching pups during the early postpartum period by placing a perforated partition between the dams and pups, and if the pups are then removed, the dams will not sustain their responsiveness to the pups at maternal tests 10 days later. These data suggest that for maternal responsiveness to be sustained, dams must receive close proximal contact with pups during their initial mother-litter interactions, allowing both chemosensory and somatosensory stimulation. The importance of chemosensory learning is suggested by some recent experiments showing that at test 10 days postpartum, dams respond preferentially to pups scented with an odor earlier paired with the pups as opposed to another familiar, but non-pup-associated scent (Malenfant, Barry, \& Fleming, 1991). Moreover, in a two-choice preference test, dams will develop a preference for the scents previously associated with pups. These data indicate that dams learn about odor characteristics of the pups and respond to those characteristics at "retention" testing.

However, experience of pup odors is not sufficient to sustain responsiveness. Odor cues must be paired with somatosensory stimulation of the dam's perioral and/or ventral regions. If, during the 1-h experience phase, dams are unable to experience perioral or ventral stimulation when they mouthe pups or when pups root for the teats, at test 10 days later dams respond as though they have never interacted with the pups before (Morgan, Fleming, \& Stern, 1992).

Since both the maternal experience effect and the acquisition of a CPP, with pups used as reinforcers, depend on interaction with pups, it is likely that the same sensory qualities or experiences that sustain maternal learning also confer reinforcing properties to the pups. In the present study, we tested this assumption by testing dams in a CPP task after a variety of chemosensory and somatosensory desensitizations such that they could not smell or feel pups while interacting with them during the pup-box pairings.

In the first study, we tested postpartum dams for CPP, using pups presented in a perforated Plexiglas cube, so that the dam could hear, see, and smell pups from a distance but could not receive proximal chemosensory or somatosensory stimulation. In the second study, we investigated the contribution to the CPP of main chemosensory stimulation by testing dams who were rendered temporarily anosmic by the intranasal application of zinc sulfate, a solution that destroys the peripheral epithelial receptor cells in the nose (Alberts \& Galef, 1971; Mayer \& Rosenblatt, 1993). In the third study, we explored the effects of desensitizing the dam's perioral or ventral regions during the training phase. This was accomplished by the application of a local topical anesthetic to either the perioral region around the mouth or the dam's ventrum.

\section{GENERAL METHOD}

\section{Animals and Housing}

The animals were Sprague-Dawley female rats, $60-90$ days of age, randomly selected from a stock bred at Erindale College. They were housed in plastic cages $(22 \times 44 \times 15 \mathrm{~cm})$, with Purina rat chow and water available ad lib and maintained under a $12: 12-\mathrm{h}$ illumination cycle (lights on at $0800 \mathrm{~h}$ ), at a room temperature of $20^{\circ}-22^{\circ} \mathrm{C}$.

\section{Apparatus}

The CPP apparatus consisted of two white PVC (polyvinyl chloride) boxes $(22 \times 40 \times 30 \mathrm{~cm})$. Horizontally and vertically striped environments were created within the CPP boxes by using electrical tape spaced $2 \mathrm{~cm}$ apart, allowing for an equal ambient illumination in the two boxes. In addition, to provide a different texture to the two environments, the horizontal box had a Plexiglas floor with equally spaced perforations (spaced $1 \mathrm{~cm}$ apart). The vertical environment had a smooth Plexiglas floor. To further distinguish the two environments, the lid to the vertical box had black electrical tape spaced at $1-\mathrm{cm}$ intervals and oriented at right angles to the vertical stripes. A complete CPP set-up consisted of one horizontal box, one vertical box, and one nonpatterned center section $(22 \times 10 \times 30 \mathrm{~cm})$ between them. The dimensions of the completed CPP apparatus were $22 \times 89 \times 30 \mathrm{~cm}$. On exposure days, the dividers between the boxes were down and only the boxes were used. On test days, the dividers were removed, allowing the dam unimpeded access to both boxes.

\section{Procedures}

All females gave birth and retained their pups for $24 \mathrm{~h}$; the day of birth was designated Day 0 . On Day 1 , pups were removed and given to another lactating donor mother. Experimental females were separated from pups for the next $23 \mathrm{~h}$, until Day 2.

On Day 2, after $23 \mathrm{~h}$ of pup deprivation, animals were started in the CPP regime.

Exposure phase. On Days 2 and 4, one half of the animals in each group were exposed first to the vertical box plus pups and one half to the horizontal box plus pups. On Days 3 and 5, animals initially exposed to the horizontal box in association with pups were exposed to the vertical box, with no pups present; similarly, animals initially exposed to the vertical box with pups on Days 2 and 4 were exposed to the horizontal box with no pups on Days 3 and 5. On all 4 days, females were placed into the boxes for a 1 -h period. On Pup Days 2 and 4, a litter of six 1- to 3-day-old pups was placed into the box into the wall side corner, away from the side of the center connector. On Days 3 and 5, females were placed alone into boxes; however, they were given six 1- to 3-day-old pups in their home cages $1-2 \mathrm{~h}$ after the end of the exposure phase. In this way, on each day at the time of box exposure, dams were between 21 and $23 \mathrm{~h}$ pup deprived.

Behavioral observations. On Days 2 and 4, during box-pup pairings, the behavior of the dam was observed and recorded, using a one-zero time-sampling procedure for a 1-min period every 10 min over the 1 -h period (spotcheck). The following behaviors were recorded: crouch posture, whereby the female adopted an arched position over the pups, giving them direct access to her ventral teat region. The crouch postures included hovering, when the dam arched her body over the pups but was also engaged in puplicking and occasional self-grooming; a low crouch, when the dam exhibited a low dorsal arch but the pups were all underneath; and a high crouch, when the dam assumed a high dorsal arch, immobile with back legs splayed and head up, and with at least some pups attached to the teats. Other recorded behaviors included pup licking, which occurred when the dam licked the pups either in the anogenital region or over the general body; pup retrieval was also recorded, but was found not to occur in this context since the boxes were novel and the dams had not constructed nest sites in them.

Test phase. On Day 6, the divider between the two patterned boxes and the center connector box was removed and dams were placed into the center connector box, giving them free access to the two boxes. A computer-based event recorder (S\&K, Norpark, Toronto, $\mathrm{ON}$ ) kept a continuous record of time spent in each of the 
two patterned boxes and the center box, as well as of all entries into each box. Tests lasted $10 \mathrm{~min}$.

Data Analysis. To evaluate maternal responding in the boxes, the number of spot checks in which the different behaviors were observed were computed, and groups were compared on these frequencies by using $t$ tests or analyses of variance. To evaluate CPP, two measures were computed. One included the proportion of the total observation time (or total time in the two boxes) animals spent in the pup-paired box; the $t$ test was used to compare pairs of groups. The other established a preference for each animal in terms of the box in which the dam spent greater than .50 of the total time spent in two boxes, combined. Binomial tests were computed separately on individual groups, to determine whether the percent of animals showing a preference within the different groups deviated significantly from chance. Comparisons between groups of the percent of animals showing a preference were made with Fisher's exact probability test. Because of the directional predictions, differences were considered significant with a $p<.05$, one-tailed.

\section{STUDY 1 \\ Effects of Distal Pup Exposure on the Development of CPP}

\section{Method}

Apparatus. To prevent dams' physical contact with pups, pups were placed into a clear Plexiglas cube $(10 \times 10 \times 8.5 \mathrm{~cm})$, with perforated sides and top and a solid floor.

Procedure. Two groups were tested, an interaction group ( $n=$ $12)$ and a distal group $(n=12)$ group. The animals in the interaction group underwent all procedures described above. On Days 2 and 4, the animals in the distal group were exposed to six 1 - to 3-day-old pups that had been placed into a perforated Plexiglas cube. The cube was placed into the far corner of the box for a 1-h period, opposite to the central divider. To ensure that females were exposed to pups at least once daily and to ensure that at the time of test females were always $21-23 \mathrm{~h}$ pup deprived, on Days 3 and 5 of the CPP training box (days without pups in boxes), the dams were given pups in their home cage for a 1-h period starting 1-2 $\mathrm{h}$ after the end of box exposure.

Behavioral observations. Since the distal animals did not have direct access to pups in the boxes, proximal maternal behaviors (crouching, licking, etc.) could not occur. In this instance, observations at each spot check included the location of the female in relation to the pups in the cube, whether the dam was on top of the cube, (sitting or lying on the cube), positioned next to the cube, or sniffing the cube. Also recorded were all attempts to move or open the cube.

\section{Results}

Exposure. During the first exposure day, $80 \%$ of the distal group showed exploratory sniffing of the cube; by the 2 nd exposure day, none did so. In contrast, on the 1 st exposure day only $30 \%$ of the animals stood or lay down on top of the box, whereas by the 2 nd exposure day $80 \%$ did so. In comparison, in the interaction group, all animals were observed in a crouch posture over the pups on the 2 exposure days; in fact, all the animals achieved a maternal rating of 1 or 2 , indicating that they were observed in a crouch posture over the pups on the majority of spot checks on at least 1 exposure day. In addition, across the 2 exposure days, $50 \%$ of the animals were observed licking the pups.
Test. As shown in Figures $1 \mathrm{~A}-1 \mathrm{~B}, 92 \%$ of the interaction group preferred the pup-paired side (binomial, $p<.003$ ) whereas only $50 \%$ of the distal group did so (n.s.). This difference was highly significant $\left[\chi^{2}(1)=\right.$ $4.8, p<.01]$. Moreover, the proportion of time spent on the pup-associated side was significantly higher in the interaction group $[t(20)=-1.75, p<.05]$.

\section{Discussion}

These results indicate that in order for pups to serve as reinforcing stimuli, the dam must actually interact with them. Exposure to pups from a distance, involving combined distal chemosensory, visual, and auditory stimulation is simply not adequate to produce a CPP, even though animals spent time positioned over the pups on top of the cube. In the next study, we considered the role of close contact chemosensory stimulation derived when the dam nosed, licked, and crouched over pups.
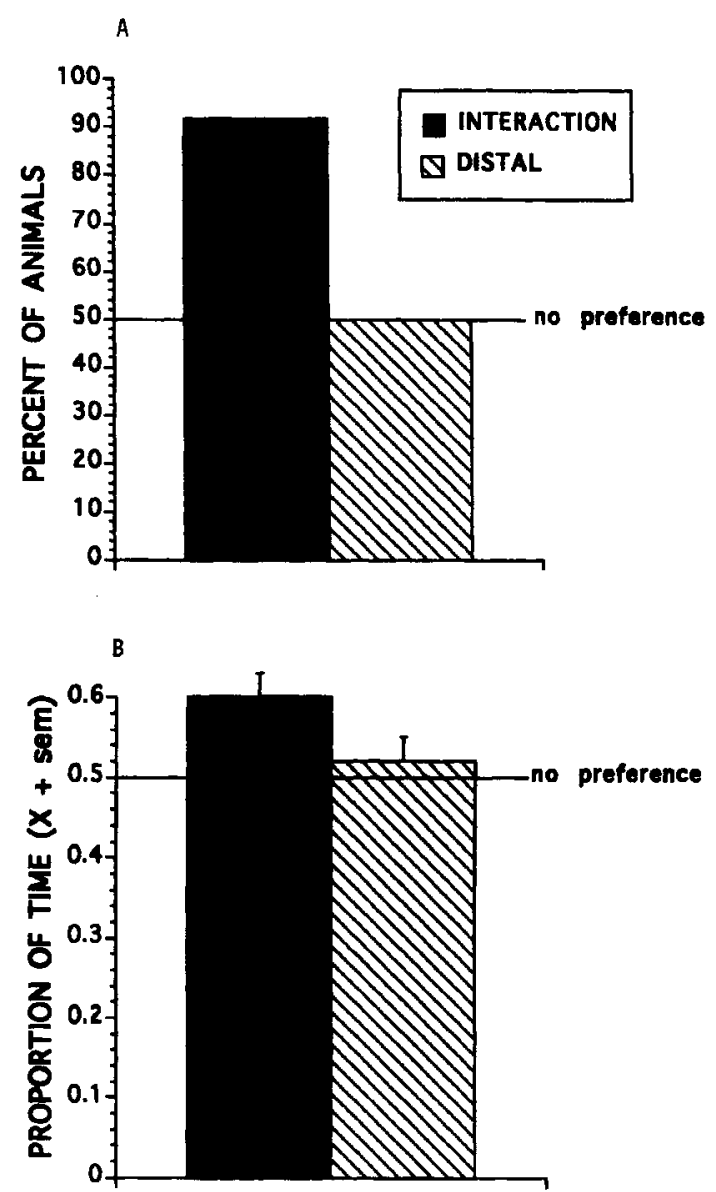

Figure 1. Animals showing a preference for the pup-paired box as a function of pup interaction during exposure: (A) percent of animals preferring pup-paired box; (B) proportion of time spent in puppaired box. A 4-day exposure regimen was used, and animals were exposed to pups for $1 \mathrm{~h}$ on each exposure day. 


\section{STUDY 2 \\ The Effects of Peripherally Induced Anosmia on CPP}

\begin{abstract}
Method
Two groups were tested, a zinc sulfate group $(n=15)$ and an air group $(n=15)$. All procedures described earlier applied in the present study, with the following additions. On Day 21 of the 22day gestation, animals in both groups were lightly anesthetized with ether and a modified 25-gauge syringe needle with a slight curvature to the tip attached to a $1-\mathrm{cc}$ syringe was gently inserted into the outer nares, until the needle tip reached the epithelial layer (approximately $12-14 \mathrm{~mm}$ from the nares' opening). A $0.05-\mathrm{ml}$ volume of a $5 \%$ zinc sulfate solution (in saline) was injected into each nose of the zinc group. An equal volume of air was injected into the air group. No saline group was included, because infusion of saline produces congestion and temporary "hypesmia" in rats (Fleming \& Rosenblatt, 1974). Following treatment of both nares, the rat was held with the head positioned below the body to allow drainage from the nares and to prevent the solution from entering the throat. After parturition, animals were all exposed to the CPP regime. This procedure produces a temporary anosmia which lasts a few days (Mayer \& Rosenblatt, 1993).
\end{abstract}

\section{Results}

Exposure. There were no group differences between anosmic and non-anosmic groups in maternal ratings or in the frequency of any of the individual maternal behaviors. Animals in both groups were maternal during the exposure phase, and $80 \%$ and $87 \%$ of the zinc and air groups, respectively, achieved a maternal rating of 1 or 2. Pup licking was observed in $53 \%$ of the zinc group and in $66 \%$ of the air group across the 2 exposure days.

Test. As can be seen in Figures $2 \mathrm{~A}-2 \mathrm{~B}$, a significant proportion of animals in the air group developed a preference for the pup-associated side $(p<.015)$, whereas only $53 \%$ of the zinc animals did so (n.s.). This difference was significant $\left[\chi^{2}(1)=3.6, p<.03\right]$. Consistent with these findings was that the two groups also differed in the proportion of time spent in the pup-paired box $[t(28)=1.73, p<.04]$.

\section{Discussion}

These results show that if dams cannot smell pups during exposure, the pups' reinforcing properties are diminished. The results are consistent with our earlier findings that during initial exposure to pups, dams develop a preference for a preexposed pup-associated odor. Thus, although the sense of smell may not be necessary for the expression of maternal behavior, it contributes to the long-term maintenance of the behavior and to the salience of pup stimulation.

\section{STUDY 3 \\ The Effects on CPP of Local Anesthetic (EMLA Cream) Applied to the Perioral or Ventral Regions}

\section{Method}

Four groups of animals were tested. For the ventral groups, the rats' ventral surfaces were treated with either anesthetic cream (EAV group, $n=10$ ) or control cream (ECV, $n=8$ ). For the perioral groups, the rat's perioral surfaces were treated with either anes- thetic cream (EAP group, $n=10$ ) or control cream (ECP group, $n=10$ ).

To obtain an anesthetizing effect on the perioral and ventral surfaces, the topical anesthesia EMLA (2.5\% lidocaine and $2.5 \%$ prilocaine, Astra Pharma Inc.) was used. The anesthetic was applied to the desired area, and it remained on the surface for $45 \mathrm{~min}$. Its anesthetic effect lasted in excess of $2 \mathrm{~h}$. For the ventral group, $2.5 \mathrm{~g}$ of EMLA was applied to the entire ventral surface from the tail to the neck and covering all nipples. For the perioral group, $1.0 \mathrm{~g}$ of EMLA was applied to the mystacial pads, the top of the nose, and the chin area. For the control groups, an equal amount of an unscented lotion (Lubriderm; Warner-Lambert Company) was used to provide a sensation of cold dampness similar to that of the topical anesthetic (EMLA).

To prevent the animals from licking off the EMLA and Lubriderm, an "Elizabethan" collar (51318, Stoelting) was placed on the animal once the topical treatments were applied. After $45 \mathrm{~min}$, the collars were removed and the excess anesthetic and lotion were cleaned off with alcohol.

For all groups, EMLA or control cream (as appropriate) were applied for a $45-\mathrm{min}$ period ending $35 \mathrm{~min}$ before the animals were placed into the CPP boxes. Treatments were applied on each of the 4 exposure days but not on the test day.

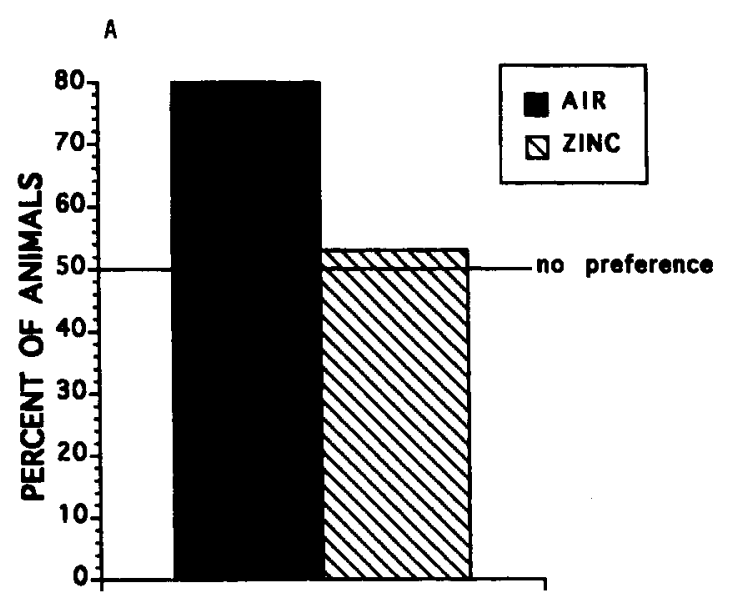

B

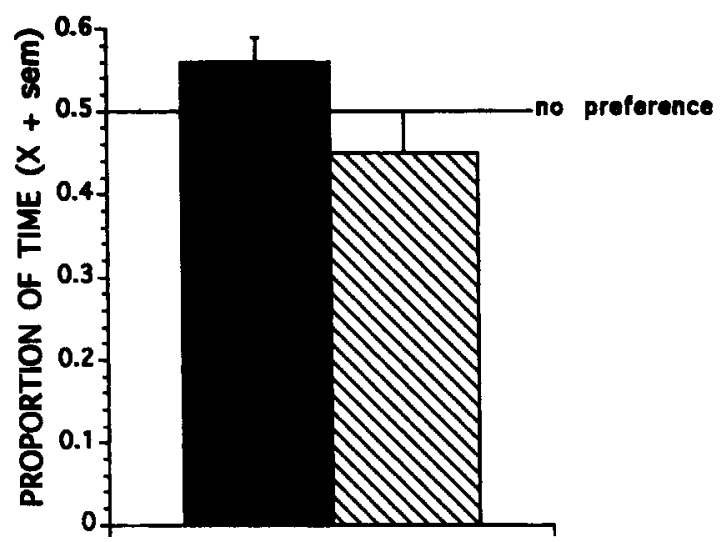

Figure 2. Animals showing a preference for the pup-paired box as a function of anosmic state during exposure: (A) percent of animals preferring pup-paired box; (B) proportion of time spent in puppaired box. A 4-day exposure regimen was used, and animals were exposed to pups for $1 \mathrm{~h}$ on each exposure day. 
To verify the anesthetic effect, all animals were given a series of tests for tactile responsivity. This involved two sets of "pinch" tests, one immediately before application of the creams and one following a $1-h$ period immediately after the creams were removed. In the "pinch" test, a muscle clamp (for the ventrum) or forceps (for the perioral region) were applied to three different areas of the ventrum or two areas of the perioral regions, and the animal's response was observed. A response was scored if the animal either "squealed" or made an abrupt movement contiguous with the pitch stimulus. In the test of ventral sensitivity, three areas were pinched: the inner side of the thigh, the midchest, and the upper chest. Tests of perioral sensitivity involved pinching both mystacial pads.

\section{Results}

Exposure. All the animals in all the groups were maternal during the exposure phase. There were no differences between groups treated with EMLA and their controls (either EAV vs. ECV or EAP vs. ECP) in maternal ratings, with maternal ratings of 1 or 2 achieved by all animals in Groups EAV, ECV, and ECP and by $90 \%$ of the animals in Group EAP. However, between the two ventrally treated groups, EAV animals were more frequently observed in a high crouch posture $\left(U=25, n_{1}=8\right.$, $n_{2}=10, p<.04$ ) but less frequently observed in a hovering position $\left(U=13, n_{1}=8, n_{2}=10, p<.01\right)$. Thus, the total time with pups under the ventral surface did not differ between the two groups. Among the periorally treated animals, there were no group differences in any maternal behaviors.

Test. The EMLA cream seems to effectively anesthetize the areas onto which it is applied. In contrast to the first pre-EMLA test, in which all animals in all groups showed a pain response, on the second test, after $50 \mathrm{~min}$ of exposure to the anesthetic, $82 \%-90 \%$ of the EMLA animals gave no response whatsoever to the pinch stimulus; in contrast, $9 \%-12 \%$ of the animals in the control groups did not respond to the pinch stimulus.

As is shown in Figures 3A-3B and 4A-4B, a significant proportion of both anesthetic control groups (Groups ECV and ECP) developed a preference for the pup-associated box (ECV, binomial, $p<.03$; ECP, binomial, $p<.05$ ); in contrast, no significant group preference was found in Group EAV or EAP (both n.s.). Comparisons between the two ventral groups and between the two perioral groups in the number of animals showing a pup-side preference showed a marginal difference for both comparisons (Fisher's test: $\mathrm{EAV}$ vs. $\mathrm{ECV}, p=.10$; EAP vs. ECP, $p=.17$ ). When the two EMLA groups were combined (EAV and EAP) and compared with the two control cream groups (ECV and ECP) in the proportion of animals showing a pup-side preference, the difference was significant $\left[\chi^{2}(1)=5.1, p<.02\right]$.

There were no significant correlations between behaviors exhibited during exposure and preference at test.

\section{GENERAL DISCUSSION}

These results indicate that if postpartum mothers are able to interact freely with pups under the influence of
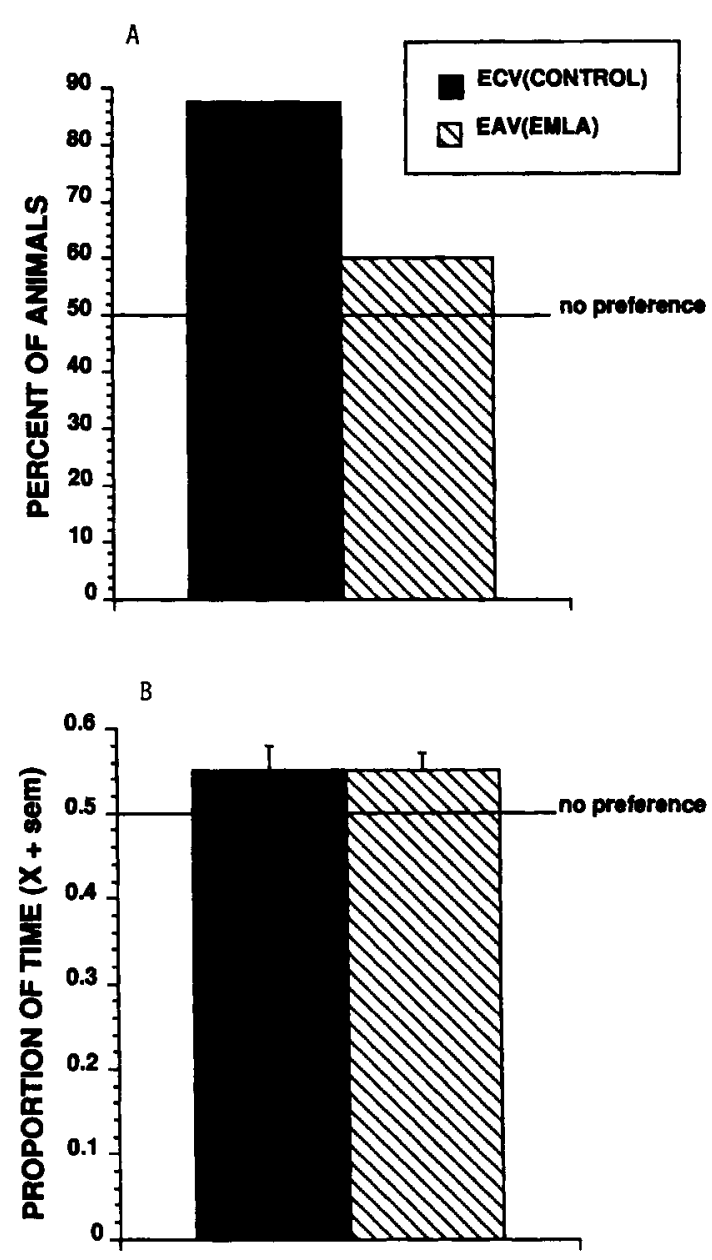

Figure 3. Animals showing a preference for the pup-paired box as a function of ventral anesthetization: (A) percent of animals preferring pup-paired box; (B) proportion of time spent in pup-paired box. A 4-day exposure regimen was used, and animals were exposed to pups for $1 \mathrm{~h}$ on each exposure day.

the parturitional hormones and be multiply stimulated by them, pups acquire reinforcing properties and are able to act as unconditioned stimuli in the formation of a CPP. These reinforcing effects function to sustain maternal responsiveness beyond the period of hormonal priming. Apparently the adequate pup stimulus is complex, involving a number of modalities; if any one sensory system is precluded during postpartum motherlitter interactions, pups are less effective reinforcers and, hence, are unable to sustain a CPP within the present temporal parameters; it is possible that if we had increased the exposure duration or the number of exposure days, the degraded pup stimulus may well have been an effective, albeit weaker, reinforcing stimulus.

The act of performing the maternal behaviors during exposure may be necessary, but it is not sufficient, for a conditioned response to develop. In fact, all desensitized groups showed quite active maternal responding, despite 


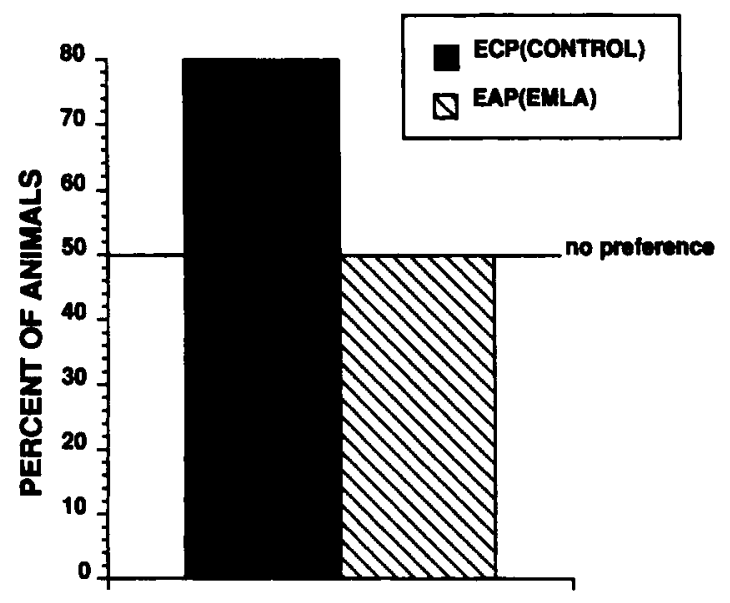

B

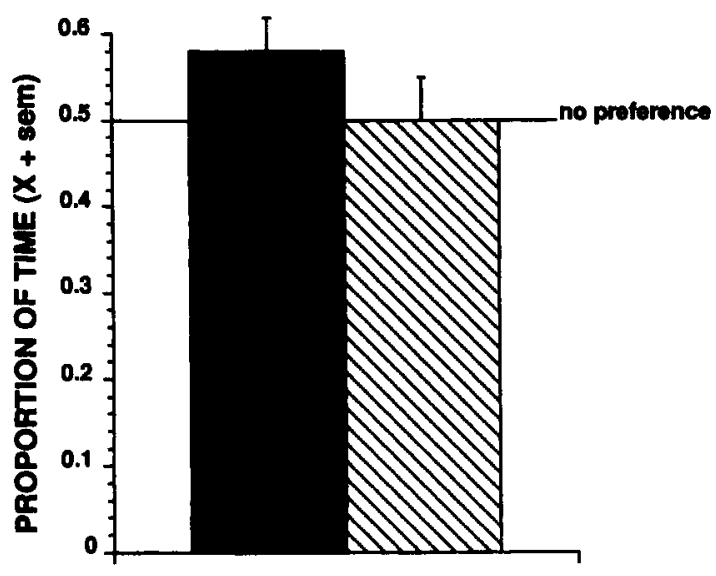

Figure 4. Animals showing a preference for the pup-paired box as a function of perioral anesthetization: (A) percent of animals preferring pup-paired box; (B) proportion of time spent in pup-paired box. A 4-day exposure regimen was used, and animals were exposed to pups for $1 \mathrm{~h}$ on each exposure day.

the fact that they were being exposed and tested in a new environment, in the absence of a permanent nest site and familiar bedding. Thus, simple expression of the behavior is not in itself highly reinforcing.

Which specific aspects of the pup stimulus complex plays a role in pup reinforcement are not known. Among the sources of pup odor cues which could influence both main and accessory olfactory systems are mother-related cues associated with milk and maternal scent glands and/or droppings in the bedding (Bauer, 1983; Leon, 1978); among pup-related cues are pup urine and feces and preputial gland secretions (Brouette-Lahlou et al., 1991). Although there is evidence that dams are attracted to maternal nest odors (Bauer, 1983; Fleming \& Luebke, 1981), whether a single odor or a complex of odors is important is not known. Since dams develop an attraction to artificial scents if these have been previously paired with pups and in fact respond differentially on reexposure to pups, depending on whether their scents are familiar or not, it is possible that reducing chemosensory sensitivity effectively blocks pup reinforcement by nonspecifically eliminating all odor stimulation. Of interest is the finding that removal of primary olfactory sensation by zinc sulfate, leaving vomeronasal function intact, nevertheless reduced pup reinforcement, since there is evidence that primary and accessory olfactory stimulation may have additive inhibitory effects on maternal behavior in virgins (Fleming, Vaccarino, Tambosso, \& Chee, 1979) and may well have additive effects in this context. It would therefore be interesting to investigate the effects of vomeronasal desensitations alone and in combination with zinc sulfate.

Our earlier observation that odors show sustained reinforcing effects only if they have been paired with ventral somatosensory stimulation (Fleming et al., 1994) is consistent with the present findings that somatosensory desensitizations reduce pup reinforcement even if puprelated odors are present. The finding that anesthetization of the dam's ventral surface resulted in lower crouch frequencies but higher frequencies of hovering suggests that the two ventrally treated groups were receiving similar amounts of ventral stimulation but of a different quality. If the ventral surface is anesthetized, the dam does not receive the kind of tactile stimulation that reflexively elicits the high crouch posture (Morgan et al., 1992; Stern et al., 1992). Instead, she compensates by spending more time hovering over pups. Somewhat surprising is the finding that perioral desensitizations were as effective in blocking a conditioned place preference as ventral desensitizations, although there was no evidence of substantially altered maternal behavior during the exposure phase in the perioral group.

One problem in the interpretation of the present data is the issue of state dependency. It is possible that since the desensitizations were in effect during each day of the exposure phase but not during the test day, the desensitized animals did not interpret the boxes experienced in the absence of tactile or odor sensitivities to be the same as boxes experienced in the presence of sensory functioning. This possibility is presently under investigation.

Although the conditioned place preference test is a useful test to assess the reinforcing properties of stimuli and has been successfully used to demonstrate the role of hormones, experience, the brain, and neurochemical systems in pup reinforcement (Fleming et al., 1994), in many ways it is an insensitive test; by its nature, it does not permit an evaluation of the course of acquisition, the intensity of the response, or the rate of extinction. With this test, it is therefore not possible to assess the relative decrements in reinforcing strength produced by the different sensory desensitizations, or possible additive effects of the combined desensitizations. For this purpose, an operant task would be more reasonable.

Despite these limitations, these CPP studies, in combination with our earlier experience studies (e.g., Morgan et al., 1992), indicate that once dams initially express maternal behavior to pups under the influence of hor- 
mones, they gain chemosensory and somatosensory experiences that enhance the pups' reinforcing value and function to sustain maternal responding beyond the early postpartum period of hormonal priming.

\section{REFERENCES}

AlLin, J. T., \& BANks, E. M. (1972), Functional aspects of ultrasound production by infant albino rats. Animal Behaviour, 20, 175-185.

AlberTs, J., \& GAlef, B. (1971). Acute anosmia in the rat: A behavioral test of a peripherally-induced olfactory deficit. Physiology \& Behavior, 6, 619-621.

BAUER, J. K. (1983). Effects of maternal state on the responsiveness to nest odors of hooded rats. Physiology \& Behavior, 20, 71-80.

BEACH, F. A., \& JAYNES, J. (1956). Studies of maternal retrieving in rats: III. Sensory cues involved in lactating female's response to her young. Behavior, 10, 104-125.

BREWSTER, J. A., \& LeON, M. (1980). Relocation of the site of motheryoung contact: Maternal transport behavior in Norway rats. Journal of Comparative \& Physiological Psychology, 94, 69-79.

BRIDGES, R. S. (1975). Long-term effects of pregnancy and parturition upon maternal responsiveness in the rat. Physiology \& Behavior, 14, 245-249.

BRIDGES, R. S. (1977). Parturition: Its role in the long term retention of maternal behavior in the rat. Physiology \& Behavior, 18, 487-490.

Brouette-Lahlou, I., Vernet-Maury, E., \& Chanel, J. (1991). Is rat-dam licking behavior regulated by pup's preputial gland secretion? Animal Learning \& Behavior, 19, 177-184.

Brouette-Lahlou, I., Vernet-Maury, E., \& VigourouX, M. (1992). Role of pups' ultrasonic calls in a particular maternal behavior in Wistar rat: Pups' anogenital licking. Behavioural Brain Research, 50, 147-154.

COHEN, J., \& BRIDGES, R. S. (1981). Retention of maternal behavior in nulliparous and primiparous rats: Effects of duration of previous maternal experience. Journal of Comparative \& Physiological Psychology, 95, 450-459.

Fleming, A. S., KorSmit, M., \& Deller, M. (1994). Rat pups are potent reinforcers to the maternal animal: Effects of experience, parity, hormones, and dopamine function. Psychobiology, 22, 44-53.

Fleming, A. S., \& LuebKe, C. (1981). Timidity prevents the nulliparous female from being a good mother. Physiology \& Behavior, 27, 863-868.

Fleming, A. S., \& RosenblatT, J. S. (1974). Olfactory regulation of maternal behavior in rats: Effects of peripherally induced anosmia and lesions of the lateral olfactory tract in pup-induced virgins. Journal of Comparative \& Physiological Psychology, 86, 233-246.

Fleming, A. S., Vaccarino, F., Tambosso, L., \& Chee, P. (1979). Vomeronasal and olfactory system modulation of maternal behavior in the rat. Science, 203, 372-374.

Kenyon, P., Cronin, P., \& KeEble, S. (1983). Role of the infraorbital nerve in retrieving behavior in lactating rats. Behavioral Neuroscience, 97, 225-269.

LEON, M. L. (1978). Filial responsiveness to olfactory cues in the laboratory rat. In D. S. Lehrman, R. A. Hinde, \& E. Shaw (Eds.), $A d$ vances in the study of behavior (pp. 117-153). New York: Academic Press.

Malenfant, S. A., Barry, M., \& Fleming, A. S. (1991). The effects of cycloheximide on olfactory learning and maternal experience effects in postpartum rats. Physiology \& Behavior, 9, 289-294.

MAYer, A. D., \& Rosenblatt, J. S. (1993). Peripheral olfactory deafferentation of the primary olfactory system in rats using $\mathrm{ZnSO}_{4}$ nasal spray with special reference to maternal behavior. Physiology \& Behavior, 53, 587-592.

Morgan, H. D., Fleming, A. S., \& Stern, J. M. (1992). Somatosensory control of the onset and retention of maternal responsiveness in primiparous Sprague-Dawley rats. Physiology \& Behavior, 51, 541-555.

Orpen, B. G., \& Fleming, A. S. (1987). Experience with pups sustains maternal responding in postpartum rats. Physiology \& Behavior, 40, 47-54.

STERN, J. M. (1989). Maternal behavior: Sensory, hormonal, and neural determinants. In F. R. Brush \& S. Levine (Eds.), Psychoendocrinology (pp. 105-226). New York: Academic Press.

Stern, J. M., Dix, L., Bellomo, C., \& Thramann, C. (1992). Ventral trunk somatosensory determinants of nursing behavior in Norway rats: 2. Role of nipple and surrounding sensations. Psychobiology, 20, 71-80.

Stern, J. M., Dix, L., Pointek, C., \& Thramann, C. T. (1990). Ventral somatosensory determinants of nursing behavior in rats: Effects of nipple loss or anesthesia. Society of Neuroscience, 16, 600 .

Stern, J. M., \& Johnson, S. K. (1989). Perioral somatosensory determinants of nursing behavior in Norway rats: Rattus norvegicus. Journal of Comparable Psychology, 103, 269-280.

Stern, J. M., \& Kolunie, J. M. (1989). Perioral anesthesia disrupts maternal behavior during early lactation in Long-Evans rats. $B e$ havioral \& Neural Biology, 52, 20-38.

(Manuscript received July 14, 1994; revision accepted for publication November $22,1994$. 